\author{
CORPUS \\ Corpus \\ $11 \mid 2012$ \\ La cooccurrence, du fait statistique au fait textuel
}

Asymétrie de la cooccurrence et contextualisation. Le rôle de la flexion casuelle dans la structuration des réseaux cooccurrentiels d'un mot-pôle en latin

Dominique Longrée et Sylvie Mellet

\title{
CpenEdition
}

Journals

Édition électronique

URL : http://journals.openedition.org/corpus/2230

DOI : $10.4000 /$ corpus.2230

ISSN : 1765-3126

Éditeur

Bases; corpus et langage - UMR 6039

Édition imprimée

Date de publication : 1 janvier 2012

ISSN : 1638-9808

Référence électronique

Dominique Longrée et Sylvie Mellet, « Asymétrie de la cooccurrence et contextualisation. Le rôle de la flexion casuelle dans la structuration des réseaux cooccurrentiels d'un mot-pôle en latin », Corpus [En ligne], 11 | 2012, mis en ligne le 18 juin 2013, consulté le 08 septembre 2020. URL : http:// journals.openedition.org/corpus/2230; DOI : https://doi.org/10.4000/corpus.2230

Ce document a été généré automatiquement le 8 septembre 2020.

(c) Tous droits réservés 


\section{Asymétrie de la cooccurrence et contextualisation. Le rôle de la flexion casuelle dans la structuration des réseaux cooccurrentiels d'un mot-pôle en latin}

Dominique Longrée et Sylvie Mellet

\section{Introduction : état des lieux et problématique}

La recherche présentée ici s'inscrit dans le cadre d'une réflexion méthodologique entamée aux JADT 2010 [Luong et al. 2010] qui a mis en évidence l'asymétrie, non seulement de la cooccurrence entre deux termes, mais aussi de la matrice qui permet de formaliser la structure globale des réseaux cooccurrentiels autour d'un mot-pôle. Nous avions alors, pour le latin, basé l'exemplification de ce nouveau paramétrage du calcul cooccurrenciel sur le dénombrement des cooccurrents d'un mot-pôle considéré et décompté sous sa forme de lemme. Or, on le sait, le latin est une langue flexionnelle et la forme prise par chaque lemme dans un énoncé est la trace de son insertion en contexte. Puisque nous considérons, avec [Mayaffre 2008], que la cooccurrence est la «forme minimale du contexte» qui contribue à construire le sens textuel, il paraît souhaitable - après ce premier travail exploratoire sur des données simplifiées qui avaient l'avantage de fournir des effectifs importants ${ }^{1}$ - de revenir maintenant à des données plus naturelles prenant en compte les indices de contextualisation que sont les marques casuelles. En effet, si « la catégorie grammaticale, par construction, influe sur l'aptitude relationnelle des unités " [Heiden \& Lafon 1998], a fortiori les marques 
morphologiques d'accord, de dépendance et de structure valencielle ont aussi un impact sur les aptitudes cooccurrentielles de chaque forme.

Nous avons fait l'hypothèse que l'asymétrie de la cooccurrence, traduite en termes de "disponibilité » et d'« énergie » cooccurrentielles, résultait des modalités de contextualisation réciproque entre deux termes [Luong et al. 2010]. Les résultats étaient originaux et leur interprétation linguistique et textuelle très stimulante. Nous voulons donc ici les étayer après avoir évalué l'impact de l'abstraction opérée par la lemmatisation sur le mot-pôle. Nous pensons que le fait de réintégrer dans le traitement un paramètre fortement conditionné par la contextualisation syntagmatique devrait avoir plus d'impact sur les «énergies » cooccurrentielles, dont nous avions montré qu'elles reflétaient plutôt la dynamique discursive des attractions lexicales, et moins d'impact sur les «disponibilités » cooccurrentielles, dont nous pensons qu'elles sont davantage conditionnées par la structure même du lexique et ses éventuels figements phraséologiques. Notre domaine d'application reste le latin, qui constitue à cet égard un champ d'investigation privilégié et pour lequel nous disposons d'outils particulièrement performants: la base de données du LASLA (Laboratoire d'Analyse Statistique des Langues Anciennes de l'Université de Liège) offre un vaste corpus de textes numérisés et annotés dans lequel chaque forme est associée à son lemme et à une analyse morphosyntaxique complète; ces fichiers sont exploitables grâce au logiciel Hyperbase Latin, développé dans le cadre du laboratoire BCL (Bases, Corpus, Langage ", UMR 7320, CNRS - Université Nice Sophia Antipolis) et qui permet à la fois l'exploration documentaire et le traitement statistique des textes.

\section{Formes casuelles et cooccurrents spécifiques}

3 Il s'agit donc de vérifier dans un premier temps que la forme casuelle d'un mot latin détermine pour une bonne part la liste de ses cooccurrents spécifiques.

Rappelons d'abord que si le rapport existant en latin entre formes casuelles et fonctions syntaxiques n'est pas univoque (une forme ne correspond pas à une fonction et une seule), il assure cependant une restriction plus ou moins importante des emplois de la forme, et réduit donc l'hétérogénéité de sa contextualisation. Il induit surtout que des formes différentes d'un même lemme présentent nécessairement des insertions différentes en contexte. Ainsi la forme du nominatif marque régulièrement le sujet (sauf pour les propositions infinitives et participiales); la forme d'accusatif est caractéristique du complément direct des verbes transitifs (même si elle marque également les sujets et attributs dans les propositions infinitives), et se rencontre après diverses prépositions, notamment celles qui introduisent des compléments de verbe exprimant la direction; la forme d'ablatif caractérise divers types de compléments circonstanciels, prépositionnels ou non, et n'apparaît que rarement comme la marque d'un argument du verbe (elle marque cependant le sujet des propositions participiales dites «ablatif absolu», propositions qui précisent une circonstance du procès décrit dans la proposition superordonnée). Examiner les cooccurrents spécifiques des différentes formes casuelles revient donc bien à prendre en compte la place de celles-ci dans la relation prédicative.

5 Deux termes vont corroborer cette hypothèse préliminaire. Il s'agit des noms POTESTAS (signifiant le « pouvoir », la "puissance ») et RATIO (signifiant le «calcul », le « compte », la « faculté de calculer », donc la « raison », le «jugement», puis ce qui 
est rationnel, donc la "théorie", la "doctrine», et enfin, par affaiblissement sémantique, la " façon », la manière »). Ces mots-pôles ont été choisis en fonction de :

6 - leur fréquence (dans la base "Latin» on dénombre 620 occurrences du lemme POTESTAS, 1403 du lemme RATIO) ;

7 - l'importance des concepts dans le corpus ;

8 - leur polysémie, qui facilite l'interprétation des variations en contexte; RATIO est cependant beaucoup plus polysémique que POTESTAS, ce qui, nous le verrons plus loin, a un impact sur nos résultats.

9 Il est en outre à noter que la distribution de ces lemmes en différentes formes casuelles s'articule essentiellement autour de trois formes du singulier, nominatif, accusatif, ablatif. Ainsi, pour le lemme POTESTAS, on dénombre 176 nominatifs singuliers, 189 accusatifs singuliers, 190 ablatifs singuliers. L'équilibre des effectifs est presque parfait. La distribution est un peu moins équilibrée pour le lemme RATIO, mais reste satisfaisante : 456 nominatifs singuliers, 293 accusatifs singuliers, 467 ablatifs singuliers. Ce sont donc les cooccurents spécifiques de ces formes que nous allons étudier dans l'ensemble de la littérature latine enregistrée au LASLA.

\subsection{Cooccurrents spécifiques de trois formes casuelles de POTESTAS}

10 On voit ci-dessous (Tableaux 1 et 2 ) le résultat de la confrontation entre les cooccurrents spécifiques de la forme de nominatif postestas et ceux de la forme d'accusatif potestatem, obtenus par la fonction Thème du logiciel Hyperbase. Celle-ci permet de sélectionner tous les paragraphes contenant la forme pivot choisie et de les constituer en sous-corpus; puis, pour chacun des mots du vocabulaire attesté dans ce sous-corpus, d'en comparer la fréquence observée avec la fréquence théorique attendue dans le cadre d'une distribution aléatoire calculée à partir du corpus entier de la base Latin. On voit ainsi les mots qui sont attirés par le mot-pôle et qui se rencontrent plus souvent qu'à leur tour dans les paragraphes contenant ce mot. La significativité de l'écart est évaluée par le calcul de l'écart réduit, dont on rappelle qu'il est significatif pour toute valeur supérieure à 2,5. Dans les tableaux ci-dessous, la première colonne donne donc la valeur de l'écart réduit, la seconde le nombre absolu d'occurrences de la forme dans le corpus entier, la troisième le nombre d'occurrences de la même forme dans le sous-corpus (extrait) et enfin la quatrième colonne donne la forme elle-même.

Tableau 1. Formes cooccurrentes spécifiques de la forme de nominatif potestas

\begin{tabular}{|l|l|l|l|}
\hline écart & corpus & texte & mot \\
\hline & & & \\
\hline 37.58 & 176 & 176 & potestas \\
\hline 8.02 & 21 & 8 & permittitur \\
\hline 6.48 & 252 & 12 & data \\
\hline
\end{tabular}




\begin{tabular}{|l|l|l|l|}
\hline 6.35 & 25 & 6 & tribunicia \\
\hline 5.32 & 13 & 4 & haerens \\
\hline 5.14 & 16 & 4 & seorsum \\
\hline 4.97 & 3253 & 31 & sit \\
\hline 4.96 & 20 & 4 & finita \\
\hline 4.87 & 22 & 4 & nequeat \\
\hline 4.84 & 23 & 4 & terminus \\
\hline 4.71 & 168 & 7 & datur \\
\hline 4.63 & 6561 & 47 & atque \\
\hline 4.60 & 1882 & 21 & esset \\
\hline 4.60 & 117 & 6 & dicendi \\
\hline 4.39 & 81 & 5 & animae \\
\hline 4.38 & 82 & 5 & iudicare \\
\hline 4.36 & 394 & 9 & romano \\
\hline 4.30 & 225 & 7 & cuique \\
\hline 4.26 & 92 & 5 & penes \\
\hline 4.25 & 156 & 6 & magistratus \\
\hline 4.15 & 102 & 5 & fieret \\
\hline 4.13 & 18 & 3 & agendi \\
\hline 4.12 & 52 & 4 & calor \\
\hline 4.10 & 574 & 10 & populo \\
\hline 4.07 & 467 & 9 & ratione \\
\hline 4.07 & 55 & 4 & 310 excipitur \\
\hline 4.02 & 21 & 30 & \\
\hline & 31 & & \\
\hline
\end{tabular}


Tableau 2. Formes cooccurrentes spécifiques de la forme d'accusatif potestatem

\begin{tabular}{|c|c|c|c|}
\hline écart & corpus & texte & mot \\
\hline 37.58 & 189 & 189 & potestatem \\
\hline 10.38 & 16 & 11 & tribuniciam \\
\hline 6.20 & 944 & 21 & populi \\
\hline 6.15 & 255 & 12 & plebis \\
\hline 5.70 & 13830 & 102 & cum \\
\hline 5.55 & 44 & 6 & necis \\
\hline 5.09 & 66 & 6 & factam \\
\hline 4.90 & 868 & 16 & romani \\
\hline 4.72 & 271 & 9 & legem \\
\hline 4.68 & 93 & 6 & uestram \\
\hline 4.63 & 150 & 7 & iudicia \\
\hline 4.60 & 8 & 3 & ferentibus \\
\hline 4.58 & 220 & 8 & summam \\
\hline 4.58 & 156 & 7 & magistratus \\
\hline 4.56 & 769 & 14 & fieri \\
\hline 4.46 & 914 & 15 & tempore \\
\hline 4.31 & 12 & 3 & adeundi \\
\hline 4.30 & 190 & 7 & potestate \\
\hline 4.28 & 129 & 6 & pompeio \\
\hline 4.26 & 7367 & 55 & esse \\
\hline 4.25 & 13 & 3 & iudicaretur \\
\hline 4.23 & 80 & 5 & habeant \\
\hline 4.20 & 14 & 3 & occidendi \\
\hline 4.15 & 574 & 11 & populo \\
\hline
\end{tabular}




\begin{tabular}{|l|l|l|l}
\hline 4.08 & 5792 & 45 & de
\end{tabular}

On constate que les deux listes sont loin d'être identiques, à la fois par la présence de cooccurrents différents et par des rangs différents pour les cooccurrents identiques.

12 Il en va de même quand on compare les cooccurrents spécifiques de la forme de nominatif postestas (Tableau 1) et ceux de la forme d'ablatif potestate (Tableau 3).

Tableau 3. Formes cooccurrentes spécifiques de la forme d'ablatif potestate

\begin{tabular}{|l|l|l|l|}
\hline écart & corpus & texte & mot \\
\hline & & & \\
\hline 37.58 & 190 & 190 & potestate \\
\hline 9.65 & 27959 & 215 & in \\
\hline 7.30 & 672 & 21 & nostra \\
\hline 5.41 & 389 & 12 & imperio \\
\hline 5.26 & 1469 & 22 & sua \\
\hline 5.02 & 308 & 10 & uestra \\
\hline 4.88 & 189 & 8 & potestatem \\
\hline 4.73 & 857 & 15 & senatus \\
\hline 4.67 & 25 & 4 & tribunicia \\
\hline 4.49 & 944 & 15 & populi \\
\hline 4.47 & 613 & 12 & facta \\
\hline 4.30 & 276 & 8 & auctoritate \\
\hline 4.24 & 574 & 11 & populo \\
\hline 4.23 & 14 & 3 & dicione \\
\hline 4.15 & 394 & 9 & romano \\
\hline 3.95 & 56 & 4 & prout \\
\hline 3.87 & 23 & 3 & mittuntur \\
\hline 3.82 & 5792 & 42 & de \\
\hline 3.78 & 1107 & 14 & tua \\
\hline 3.78 & 26 & 3 & reliquorum \\
\hline
\end{tabular}




\begin{tabular}{|l|l|l|l|}
\hline 3.72 & 617 & 10 & bona \\
\hline 3.71 & 868 & 12 & romani \\
\hline 3.70 & 1882 & 19 & esset \\
\hline 3.70 & 73 & 4 & habemus \\
\hline 3.70 & 29 & 3 & positam \\
\hline
\end{tabular}

13 Ces listes des cooccurrents spécifiques de chacune des trois formes de POTESTAS présentent certes des convergences: on retrouve, dans les trois listes, des formes de tribunicius (« [puissance] tribunicienne »), de populus et de Romanus (« peuple romain »), de magistratus («magistrat »). Mais on relève aussi des divergences non négligeables; par exemple, quand on descend un peu plus loin dans la liste des cooccurrents spécifiques de la forme d'accusatif, on y trouve des termes renvoyant à la puissance impériale romaine, y compris dans ses modalités guerrières : castris « camp » $(z=3,84)$, pugnandi « de combattre » $(3,81)$, copiis «troupes» $(3,54)$, colonias « colonies » $(3,50)$, uectigalis « impôts » $(3,36)$, belli « guerre » $(3,0)$ - termes que l'on ne retrouve pas dans la liste des cooccurrents spécifiques de la forme de nominatif. A celle-ci, en revanche, on trouve associés des termes tels que " maittres ", « rois ", " règne ", «force ». On voit bien, à travers ce simple exemple, que l'on est pourtant toujours bien dans la cooccurrence thématique, pas dans la rection grammaticale ou la contrainte distributionnelle.

Toutefois, on peut se demander si le calcul des cooccurrents spécifiques appréhendés sous formes graphiques n'introduit pas un biais: il est évident, en effet, que le cooccurrent spécifique de l'accusatif potestatem sera plus facilement un accusatif du type nostram («notre pouvoir ») ou tribuniciam («la puissance tribunicienne ») que toute autre forme déclinée de ces adjectifs. Et d'autres phénomènes moins triviaux pourraient impacter subrepticement nos résultats. On a donc refait le calcul d'attraction spécifique sur le texte réduit à ses lemmes, en prenant toujours pour pivot chacune des trois formes de POTESTAS. Voici les résultats.

Tableau 1 bis. Lemmes cooccurrents spécifiques de la forme de nominatif potestas ${ }^{2}$

\begin{tabular}{|l|l|l|l|}
\hline écart & corpus & texte & mot \\
\hline & & & \\
\hline 37.58 & 620 & 181 & potestas \\
\hline 6.37 & 333 & 13 & permitto \\
\hline 5.95 & 36 & 6 & seorsvm1 \\
\hline 5.77 & 74 & 7 & tribvnicivs2 \\
\hline 5.25 & 385 & 11 & magistratvs \\
\hline
\end{tabular}




\begin{tabular}{|l|l|l|l|}
\hline 5.05 & 80 & 6 & decemvir \\
\hline 4.72 & 6441 & 47 & atqve1 \\
\hline 4.53 & 918 & 14 & ivdico \\
\hline 4.52 & 359 & 9 & motvs \\
\hline 4.42 & 2477 & 24 & popvlvs1 \\
\hline 4.24 & 1779 & 19 & lex \\
\hline 4.24 & 93 & 5 & penes \\
\hline 4.00 & 1927 & 19 & fio \\
\hline 4.00 & 374 & 8 & anima \\
\hline 3.95 & 8299 & 51 & cvm3 \\
\hline 3.92 & 5790 & 39 & de \\
\hline 3.78 & 1250 & 14 & ivs1 \\
\hline 3.74 & 16929 & 87 & is \\
\hline 3.73 & 2461 & 21 & vos \\
\hline 3.69 & 83 & 4 & intercedo1 \\
\hline 3.66 & 1473 & 15 & ivdicivm \\
\hline 3.64 & 8533 & 50 & possvm1 \\
\hline 3.64 & 725 & 10 & deniqve \\
\hline 3.64 & 159 & 5 & finio \\
\hline 3.60 & 165 & 5 & qvisnam \\
\hline 3.58 & 93 & 4 & terminvs \\
\hline
\end{tabular}

Tableau 2 bis. Lemmes cooccurrents spécifiques de la forme d'accusatif potestatem

\begin{tabular}{|l|l|l|l|}
\hline écart & corpus & texte & mot \\
\hline & & & \\
\hline 37.58 & 620 & 200 & potestas \\
\hline 9.37 & 74 & 14 & tribvnicivs2 \\
\hline
\end{tabular}




\begin{tabular}{|l|l|l|l|}
\hline 8.78 & 2477 & 47 & popvlvs1 \\
\hline 8.60 & 112 & 14 & redigo \\
\hline 7.84 & 21 & 8 & cvriatvs \\
\hline 7.17 & 1779 & 33 & lex \\
\hline 7.01 & 2298 & 37 & romanvsa \\
\hline 6.87 & 8299 & 79 & cvm3 \\
\hline 6.24 & 16929 & 124 & is \\
\hline 6.12 & 385 & 14 & magistratvs \\
\hline 5.64 & 5204 & 51 & habeo \\
\hline 5.63 & 333 & 12 & permitto \\
\hline 5.62 & 7149 & 63 & facio \\
\hline 5.54 & 502 & 14 & plebs \\
\hline 5.42 & 83 & 7 & intercedo1 \\
\hline 5.33 & 12234 & 90 & svm2 \\
\hline 5.16 & 505 & 13 & tribvnvs \\
\hline 5.08 & 9002 & 70 & svi1 \\
\hline 4.93 & 657 & 14 & pompeivs@ \\
\hline 4.90 & 123 & 7 & vectigal \\
\hline 4.70 & 10249 & 74 & vt4 \\
\hline 4.69 & 438 & 11 & decerno \\
\hline 4.45 & 918 & 15 & ivdico \\
\hline 4.37 & 1710 & 21 & senatvs \\
\hline 4.31 & 189 & 7 & creo \\
\hline 4.26 & 1498 & 19 & civitas \\
\hline
\end{tabular}

Tableau 3 bis. Lemmes cooccurrents spécifiques de la forme d'ablatif potestate \begin{tabular}{|l|l|l|l}
\hline écart & corpus & texte & mot
\end{tabular} 


\begin{tabular}{|c|c|c|c|}
\hline 37.58 & 620 & 202 & potestas \\
\hline 9.72 & 27800 & 215 & in \\
\hline 5.79 & 2477 & 32 & popvlvs1 \\
\hline 5.56 & 1000 & 19 & provincia \\
\hline 5.06 & 1314 & 20 & vester \\
\hline 5.04 & 5204 & 46 & habeo \\
\hline 5.03 & 1710 & 23 & senatvs \\
\hline 5.01 & 74 & 6 & tribvnicivs2 \\
\hline 4.98 & 16929 & 108 & is \\
\hline 4.96 & 2298 & 27 & romanvsa \\
\hline 4.92 & 6714 & 54 & svvs \\
\hline 4.90 & 411 & 11 & qvintvs@ \\
\hline 4.80 & 269 & 9 & brvtvs@ \\
\hline 4.51 & 30 & 4 & cvratio \\
\hline 4.47 & 116 & 6 & praeditvs \\
\hline 4.26 & 137 & 6 & designo \\
\hline 4.21 & 85 & 5 & decimvs@ \\
\hline 4.17 & 1473 & 18 & ivdicivm \\
\hline 4.10 & 1230 & 16 & consvl \\
\hline 4.09 & 731 & 12 & avctoritas \\
\hline 4.09 & 48 & 4 & staienvs@ \\
\hline 4.05 & 18 & 3 & caepio@ \\
\hline 4.00 & 428 & 9 & gallia@ \\
\hline 3.95 & 56 & 4 & provt \\
\hline 3.89 & 39989 & 202 & svm1 \\
\hline 3.86 & 463 & 9 & emo \\
\hline
\end{tabular}




\begin{tabular}{|l|l|l|l|}
\hline 3.82 & 5790 & 42 & de \\
\hline 3.82 & 64 & 4 & proconsvl \\
\hline 3.79 & 283 & 7 & accvsator \\
\hline 3.76 & 2160 & 21 & respvblica \\
\hline 3.72 & 1273 & 15 & imperivm \\
\hline
\end{tabular}

Comme on pouvait s'y attendre, ce nouveau calcul sur les lemmes dégrade de rang les mots rares dont peu de formes sont attestées en cooccurrence avec la forme pivot. A l'inverse sont remontés dans la liste les mots fréquents dont plusieurs formes casuelles sont utilisées dans l'environnement de la forme pivot; par exemple, avec le nominatif potestas : magistratus « le magistrat", decemuir « le decemvir ", iudico " juger ", populus « le peuple ", lex « la loi »; avec l'accusatif potestatem : redigo " réduire », habeo « avoir ", curiatus « curiate ", uectigal « l'impôt »; avec l'ablatif potestate : prouincia « la province », populus romanus « le peuple romain », consul et proconsul « le consul » et « le proconsul », Gallia « la Gaule ». Les champs thématiques se précisent donc.

\subsection{Cooccurrents spécifiques de trois formes casuelles de RATIO}

Les mêmes tests appliqués à l'environnement des mêmes formes casuelles du lemme RATIO donnent des résultats comparables : le paramètre morphosyntaxique a bien un impact sur la liste des cooccurrents spécifiques de la forme, au-delà des simples phénomènes d'accord.

Ainsi les premiers verbes cooccurrents de la forme de nominatif ratio sont: sum " être ", contingo « se produire ", consto « être établi ", postulo « demander "; ceux de la forme d'accusatif rationem sont: reddo "rendre", habeo "avoir ", oportet «il faut", contingo "se produire "; et ceux de la forme d'ablatif ratione sont : possum "pouvoir", queo "pouvoir», creo "créer», cerno "voir, distinguer», perspicio "percevoir, distinguer ». Par ailleurs, la liste autour de rationem suggère un lien thématique fort avec des realia économiques (officium «la charge», pecunia «l'argent», annona «le marché », frumentum «le blé », ueneo " être vendu»). Autour de l'ablatif, on trouve de nombreux adjectifs, dus peut-être à un début de grammaticalisation de la forme ratione qui devient nom support pour les compléments adverbiaux de manière (" d'une façon semblable, dissemblable, véridique, vaine, autre, certaine »); mais aussi un réseau thématique emprunté à la philosophie naturaliste («le corps», «la nature», «les éléments primordiaux », « le soleil », « la lune », « l'air »)².

En conclusion, les réseaux cooccurrentiels autour de ces formes-pôles sont différents entre eux. Ils ne peuvent donc qu'être différents aussi de celui du lemme qui, certes, les subsume, les synthétise, mais lisse sensiblement leurs écarts et occulte les différences les plus marquées. C'est en exploitant ces différences que nous allons maintenant vérifier notre seconde hypothèse, à savoir que les différences entre les réseaux cooccurrentiels du lemme et de ses formes pourraient venir corroborer les conclusions interprétatives que nous avons avancées sur la différence entre "énergie» et « disponibilité » cooccurrentielles. 


\section{Réseaux cooccurrentiels et matrices asymétriques : le jeu du lemme et des formes}

\subsection{Rappel sur l'asymétrie de la cooccurrence}

19 Cette asymétrie est clairement perceptible, par exemple, dans le cas du syntagme voie lactée. Si, en se fondant sur les attetations fournies par la base Frantext après 1850, on rapporte le nombre d'occurrences du syntagme (295 attestations) d'une part au nombre d'occurrences de lactée (352 attestations) et d'autre part au nombre d'occurrences de voie (12 803 attestations), on constate que lactée attire très nettement voie - ou, plus exactement, consacre une très large part de ses effectifs à la cooccurrence avec voie (295/352), mais qu'en revanche voie n'attire pas lactée ou ne consacre qu'une faible part de ses occurrences à s'associer avec cet adjectif (352/12803). En d'autres mots, le réseau d'un ensemble de termes cooccurrents $[\mathrm{A}, \mathrm{B}, \mathrm{C}, \mathrm{D}, \mathrm{E}]$ s'organise de manière différente selon que, dans la matrice carrée des cooccurrences, on rapporte le nombre des cooccurrences $\mathrm{AB}, \mathrm{AC}, \mathrm{AD}, \mathrm{AE}$ soit à l'effectif de $\mathrm{A}$, soit respectivement aux effectifs de $\mathrm{B}$, de $\mathrm{C}$, de $\mathrm{D}$ et de $\mathrm{E}$.

On propose dès lors de distinguer pour chaque mot son énergie et sa disponibilité cooccurrentielles.

21 Ainsi, si l'on divise le nombre de cooccurrences de $\mathrm{D}$ avec $\mathrm{A}\left(\mathrm{D}^{*} \mathrm{~A}\right)$, avec $\mathrm{B}\left(\mathrm{D}^{*} \mathrm{~B}\right)$, avec $\mathrm{C}$ $\left(D^{*} \mathrm{C}\right)$, avec $\mathrm{E}\left(\mathrm{D}^{*} \mathrm{E}\right)$, etc. par l'effectif de $\mathrm{D}$, on mesure l'énergie cooccurentielle de $\mathrm{D}$, à savoir la part de ses effectifs que D consacre à chacun des autres termes du réseau.

En revanche, si on divise ce même nombre de cooccurrences de D avec A, B, C, E etc., respectivement par le nombre d'occurrences de $\mathrm{A}, \mathrm{B}, \mathrm{C}$ ou $\mathrm{E}$, on mesure alors quelle proportion des effectifs de A, B, C ou E, l'élément D mobilise ou, en d'autres termes, la part des effectifs des cooccurrents qu'il accueille dans son champ cooccurrentiel. Dans le deuxième cas, on parlera de sa disponibilité à être en cooccurrence avec les autres termes.

Etendue à l'ensemble des termes cooccurrents, cette distinction permet de construire une matrice carrée asymétrique dans laquelle chaque élément a un profil en ligne (énergie) et un profil en colonne (disponibilité). 
Tableau 4. Matrice cooccurentielle asymétrique (en bleu, la mesure de l'énergie cooccurrentielle du terme $\mathrm{D}$, en jaune la mesure de sa disponibilité cooccurrentielle)

\begin{tabular}{|c|c|c|c|c|c|}
\hline & $\mathbf{A}$ & $\mathbf{B}$ & $\mathbf{C}$ & $\mathbf{D}$ & $\mathbf{E}$ \\
\hline $\mathrm{A}$ & $\left(\mathrm{A}^{*} \mathrm{~A}\right) / \mathrm{A}$ & $\left(\mathrm{A}^{*} \mathrm{~B}\right) / \mathrm{A}$ & $\left(\mathrm{A}^{*} \mathrm{C}\right) / \mathrm{A}$ & $\left(\mathrm{A}^{*} \mathrm{D}\right) / \mathrm{A}$ & $\left(\mathrm{A}^{*} \mathrm{E}\right) / \mathrm{A}$ \\
\hline $\mathrm{B}$ & $\left(\mathrm{B}^{*} \mathrm{~A}\right) / \mathrm{B}$ & $\left(\mathrm{B}^{*} \mathrm{~B}\right) / \mathrm{B}$ & $\left(\mathrm{B}^{*} \mathrm{C}\right) / \mathrm{B}$ & $\left(\mathrm{B}^{*} \mathrm{D}\right) / \mathrm{B}$ & $\left(\mathrm{B}^{*} \mathrm{E}\right) / \mathrm{B}$ \\
\hline $\mathrm{C}$ & $\left(\mathrm{C}^{*} \mathrm{~A}\right) / \mathrm{C}$ & $\left(\mathrm{C}^{*} \mathrm{~B}\right) / \mathrm{C}$ & $\left(\mathrm{C}^{*} \mathrm{C}\right) / \mathrm{C}$ & $\left(\mathrm{C}^{*} \mathrm{D}\right) / \mathrm{C}$ & $\left(\mathrm{C}^{*} \mathrm{E}\right) / \mathrm{C}$ \\
\hline $\mathrm{D}$ & $\left(\mathrm{D}^{*} \mathrm{~A}\right) / \mathrm{D}$ & $\left(\mathrm{D}^{*} \mathrm{~B}\right) / \mathrm{D}$ & $\left(\mathrm{D}^{*} \mathrm{C}\right) / \mathrm{D}$ & $\left(\mathrm{D}^{*} \mathrm{D}\right) / \mathrm{D}$ & $\left(\mathrm{D}^{*} \mathrm{E}\right) / \mathrm{D}$ \\
\hline $\mathrm{E}$ & $\left(\mathrm{E}^{*} \mathrm{~A}\right) / \mathrm{E}$ & $\left(\mathrm{E}^{*} \mathrm{~B}\right) / \mathrm{E}$ & $\left(\mathrm{E}^{*} \mathrm{C}\right) / \mathrm{E}$ & $\left(\mathrm{E}^{*} \mathrm{D}\right) / \mathrm{E}$ & $\left(\mathrm{E}^{*} \mathrm{E}\right) / \mathrm{E}$ \\
\hline
\end{tabular}

Des calculs de distances et des représentations arborées permettent ensuite de mettre en évidence les regroupements ou éloignements entre les termes en fonction de ces deux paramètres: la représentation arborée sur les lignes permet de visualiser les distances entre les profils cooccurrentiels d'énergie ; la représentation arborée sur les colonnes donne à voir les distances entre les profils cooccurrentiels de disponibilité.

\subsection{Le réseau cooccurrentiel du lemme POTESTAS : énergies et disponibilités}

Plusieurs tests sur différents types de textes et dans des langues différentes [Luong et al. 2010] ont suggéré que l'arbre des disponibilités manifeste plutôt des regroupements entre termes par champs lexicaux stabilisés ou par proximités phraséologiques, tandis que l'arbre des énergies s'organise prioritairement en fonction de regroupements thématiques et notionnels contextualisés en discours. La prise en compte de formes casuelles du lemme nous offre ici l'occasion d'un test pertinent pour renforcer ces premiers résultats.

Nous allons mettre en œuvre le texte paradoxal suivant : nous allons établir la liste des lemmes cooccurrents spécifiques du lemme POTESTAS et sélectionner les 25 premiers termes de sens plein dans cette liste (substantifs, verbes, adjectifs); puis, nous allons calculer le nombre de cooccurrences de ces termes dans l'environnement d'une forme fléchie de POTESTAS, établir la matrice carrée asymétrique évoquée ci-dessus (énergies et disponibilités) et représenter la structuration de ce réseau sous forme arborée. Notre hypothèse est que, puisque le réseau cooccurrentiel (L) d'un lemme est une abstraction synthétique de l'ensemble des réseaux cooccurrentiels $\left(l_{1}, l_{2}, l_{3}, l_{4}, 1_{5}, l_{6}\right)$ des différentes formes fléchies de ce lemme, la projection de ce réseau sur l'environnement d'une forme et le calcul des affinités de comportement entre les termes du réseau dans cet environnement devraient donner des arbres de moins bonne qualité que lorsque la matrice est celle qui comptabilise les cooccurrences des termes de $\mathrm{L}$ dans l'environnement du lemme lui-même (mot-pôle qui a justement servi à constituer $\mathrm{L}$ ). 
27 Mais il est à prévoir aussi et surtout que l'arbre des énergies, s'il est vraiment représentatif de la contextualisation discursive des formes du réseau, sera mal structuré et difficilement interprétable (en raison de l'imposition forcée d'une liste de cooccurrents sur un environnement contextuel trop éloigné de celui du lemme), tandis que l'arbre des disponibilités pourrait être moins perturbé par cette manipulation et rester interprétable, dans la mesure où il donne à voir des rapprochements plus décontextualisés et éventuellement stabilisés dans le lexique de la langue (donc moins sensibles à l'insertion contextuelle manifestée par la forme casuelle).

28 2.2.1 Voyons d'abord, en guise de point de référence comparatif, comment se structure le réseau cooccurrentiel du lemme POTESTAS, appréhendé normalement dans le contexte de ce dernier. Le calcul de distance sur les colonnes (disponibilités) donne le résultat ci-dessous (figure 1), où l'on observe des regroupements phraséologiques sur les branches nord et ouest de l'arbre. A l'ouest, les regroupements sont liés à des expressions récurrentes telles que tribunus plebis intercessit, lex curiata, lex tribunicia ${ }^{4}$; au nord, on retrouve des termes intervenant dans des expressions telles que senatus decreuit populusque iussit, a senatu decretum est, prouinciae decernuntur ${ }^{5}$... Ces unités phraséologiques contextualisent ensemble les autres éléments du réseau, ce qui explique la proximité de leur profil. Sur la branche sud-est, on observe un regroupement étymologique et lexical, de mots de même racine (iudico, iudex, iudicium $\left.{ }^{6}\right)$. Avec un sème partagé, ces termes contextualisent de façon semblable tous les éléments du réseau. 


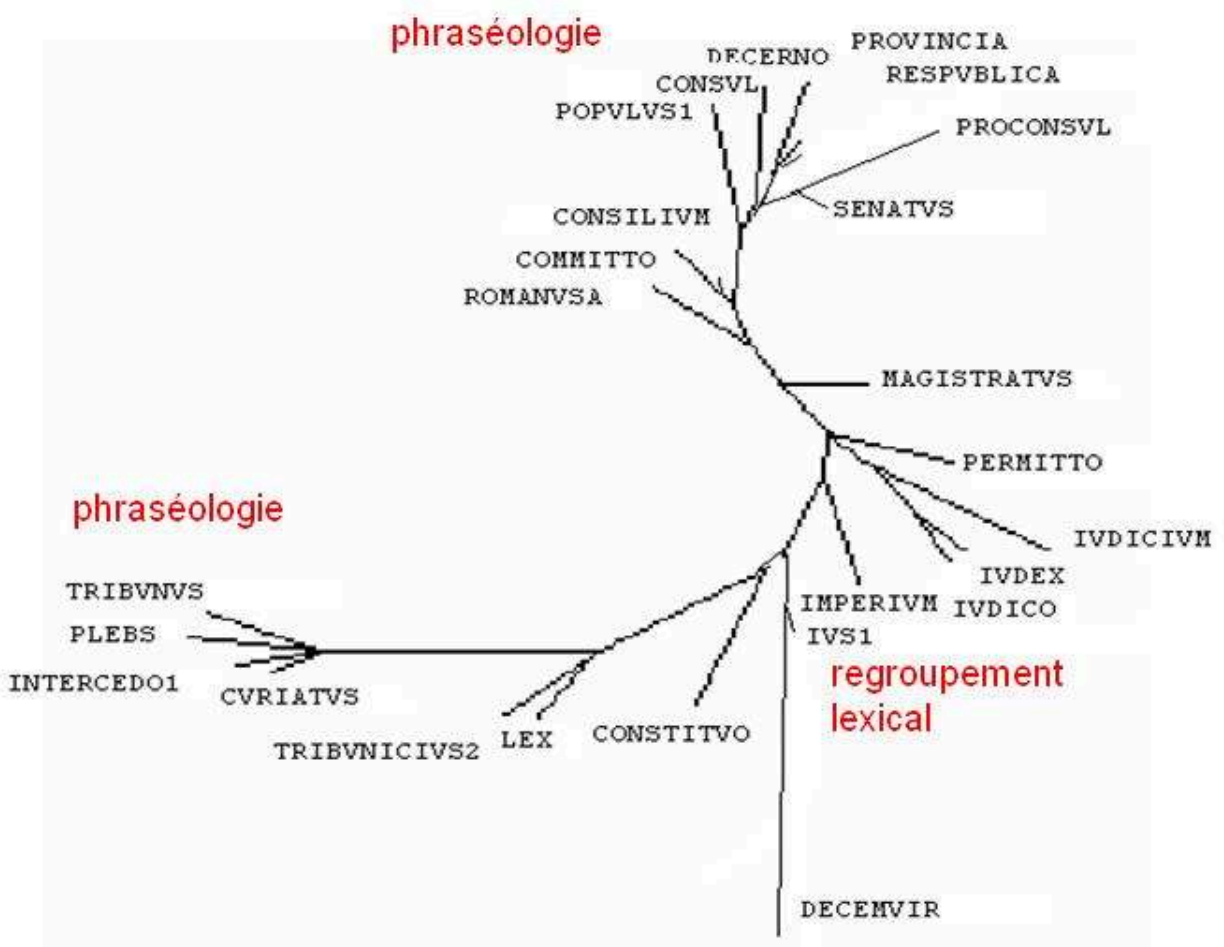

Figure 1. Arbre des disponibilités du réseau cooccurrentiel du lemme POTESTAS

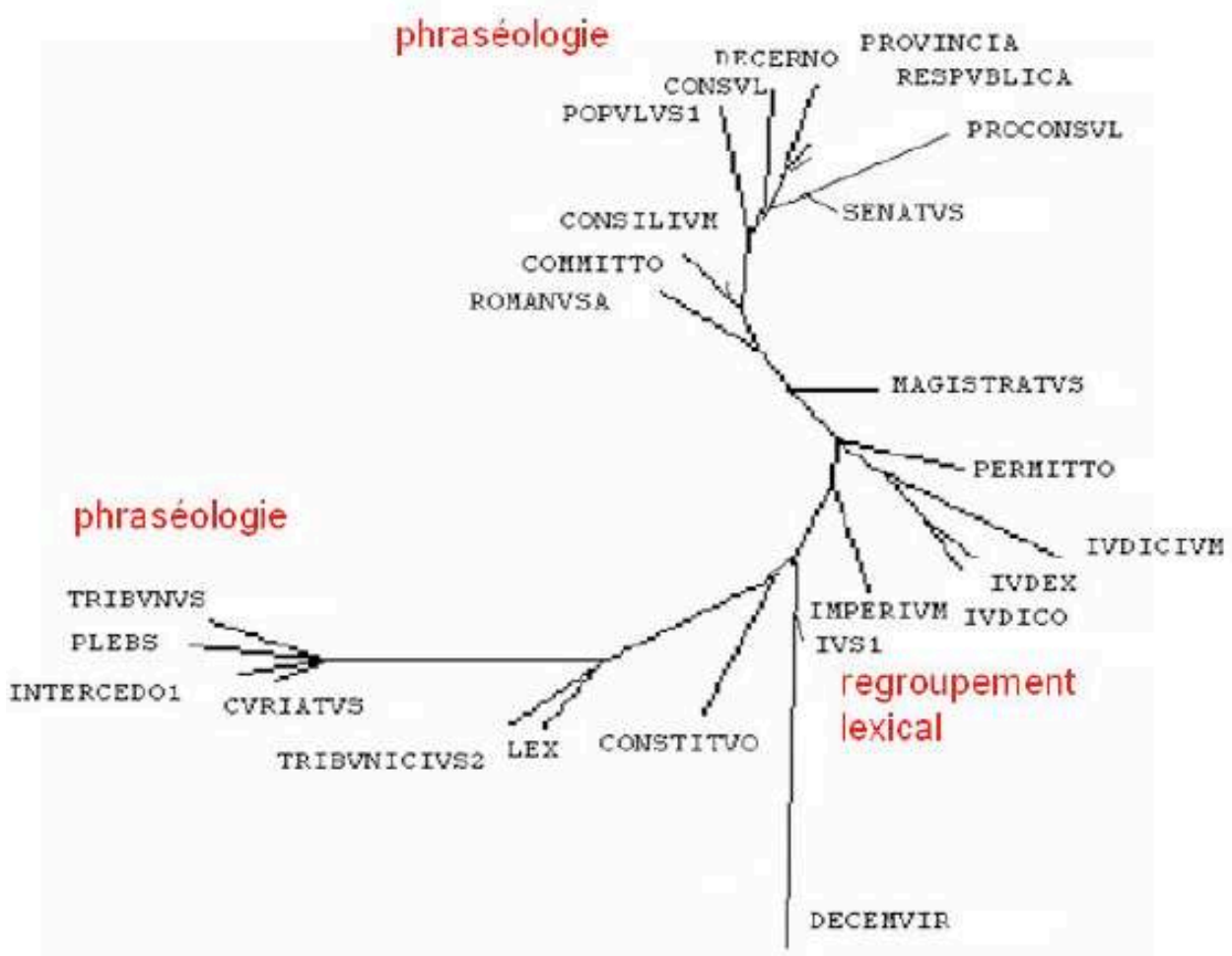

Sur l'arbre des énergies (figure 2), on note une stabilité globale des proximités entre les différents profils, mais avec néanmoins quelques modifications significatives. Sur la branche ouest, consul, constituo et lex se rapprochent de plebs, intercedo, tribunus, sur un ensemble de branches serrées formant un groupe extrêmement cohérent du point de vue référentiel et correspondant au domaine du législatif et de la politique intérieure. 
Sur la branche nord, ius se rapproche de imperium et est contextualisé par le corpus comme un élément de la puissance extérieure romaine. Sur la branche sud, le rapprochement sensible de magistratus et decemuir contribue à créer un regroupement thématique autour du juridique.

Figure 2. Arbre des énergies du réseau cooccurrentiel du lemme POTESTAS

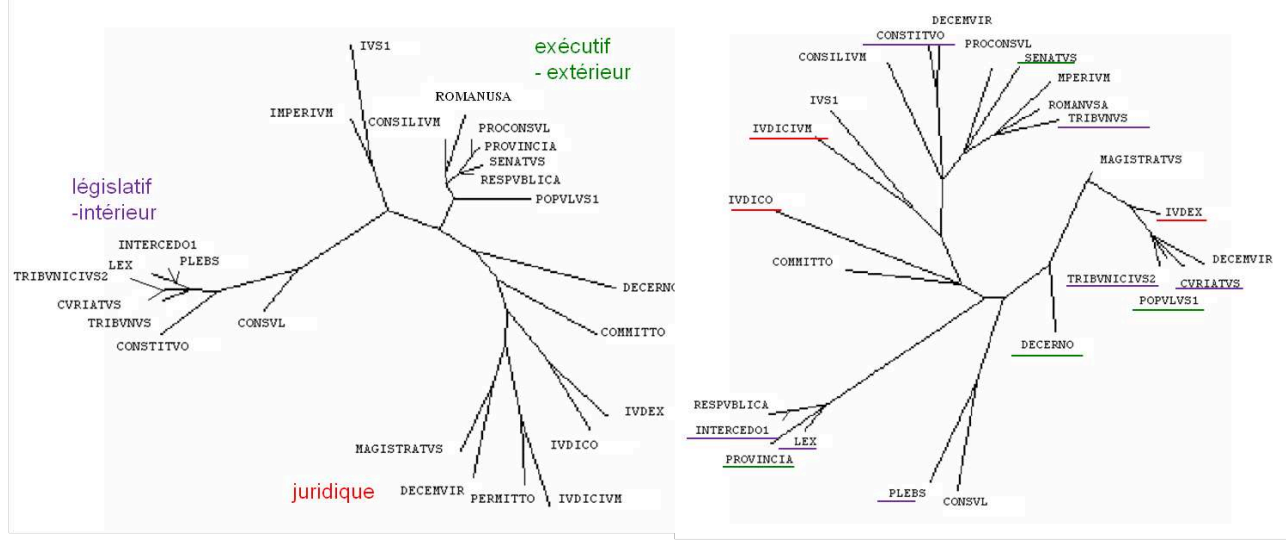

30 L'étude de ce réseau cooccurrentiel confirme donc bien que l'arbre des disponibilités (c'est-à-dire celui qui donne à voir les proximités et éloignements des termes en fonction de l'aptitude de chacun à capter telle ou telle part des effectifs de chacun des autres) manifeste plutôt des regroupements par champs lexicaux stabilisés ou par proximités phraséologiques, tandis que l'arbre des énergies s'organise prioritairement en fonction de regroupements thématiques et notionnels contextualisés.

Nous allons maintenant soumettre ce constat au test précédemment décrit.

2.2.2 Pour ce faire, nous avons repris les 25 éléments du réseau précédent et établi la matrice carrée asymétrique de leurs cooccurrences, non plus dans le contexte du lemme, mais dans le contexte de chacune des trois formes fléchies étudiées, à savoir potestas, potestatem et potestate.

Voici les arbres obtenus pour la forme d'ablatif, mis en regard de ceux construits pour le lemme (figure 3).

Arbre des disponibilités du réseau cooccurrentiel calculé autour du lemme POTESTAS (reprise de la figure 1)

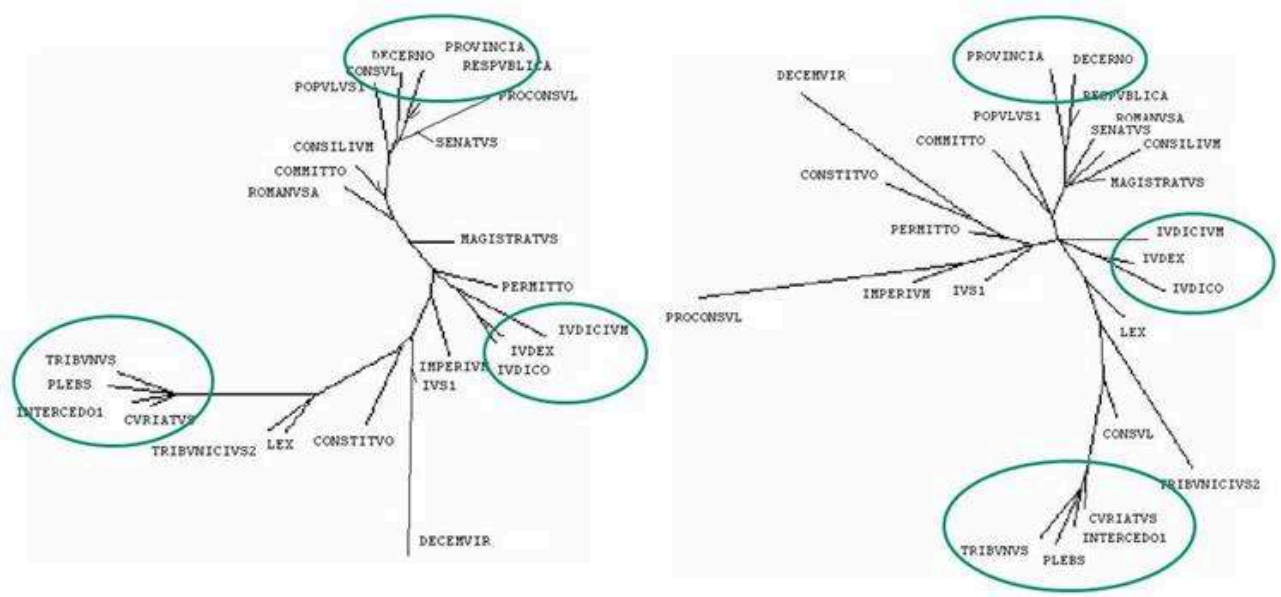


Figure 3. Arbre des disponibilités du même réseau cooccurrentiel calculé autour de la forme potestate

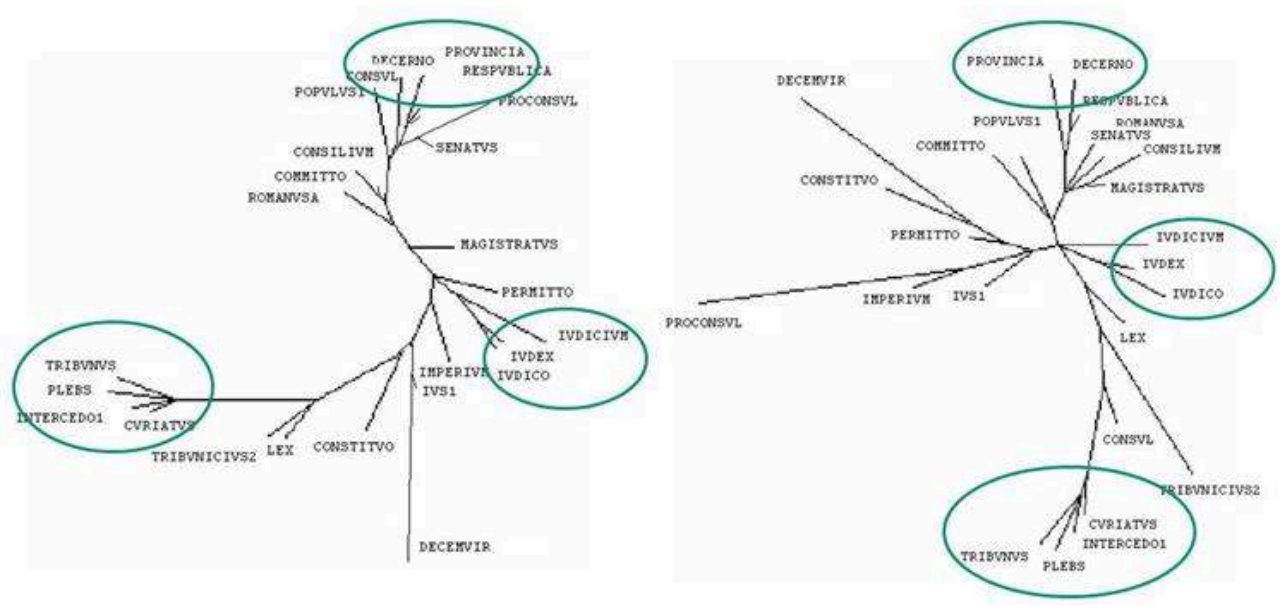

Dans cette confrontation entre l'arbre des disponibilités calculé sur les cooccurrences des 25 termes de la liste dans l'environnement du lemme et celui calculé sur les cooccurrences entre les mêmes termes dans l'environnement de la forme potestate, on voit que le second présente certes une déstructuration par rapport au premier, mais que les regroupements phraséologiques et étymologiques les plus forts se maintiennent (signalés par une ellipse: decernere prouinciam "attribuer une province [à un proconsul]; tribunus plebis intercessit « le tribun de la plèbe a posé son veto »; famille étymologique autour de la notion de jugement : iudico, iudex, iudicium).

En revanche, la même comparaison, mais portant cette fois sur les arbres des énergies montre un éclatement, non seulement des regroupements thématiques, mais même des regroupements phraséologiques ou étymologiques.

Arbre des énergies du réseau cooccurrentiel calculé autour du lemme POTESTAS (reprise de la figure 2)
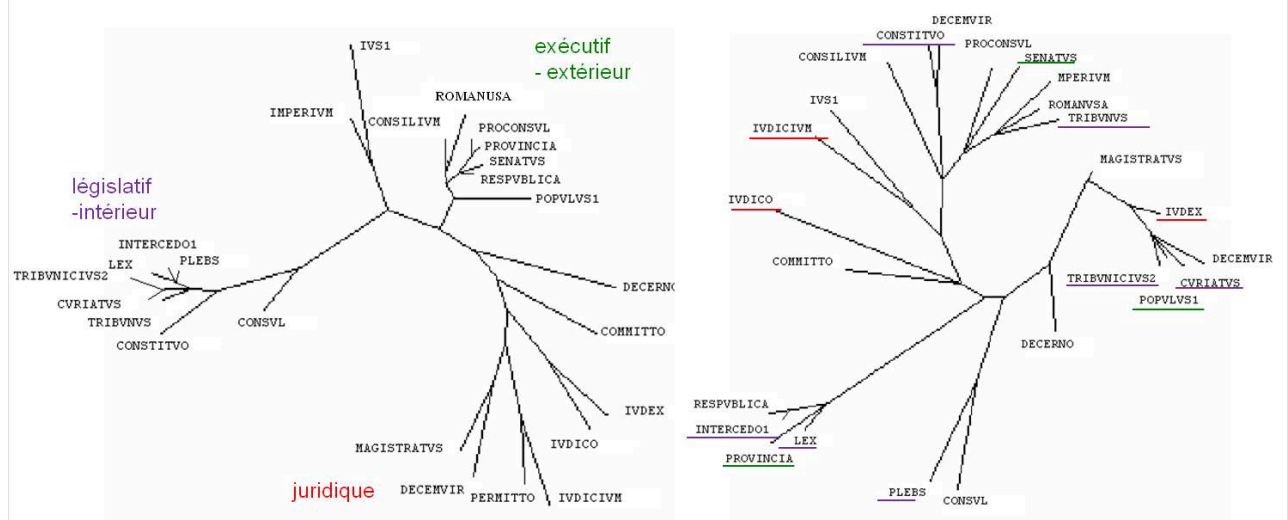
Figure 4. Arbre des énergies du même réseau cooccurrentiel calculé autour de la forme potestate

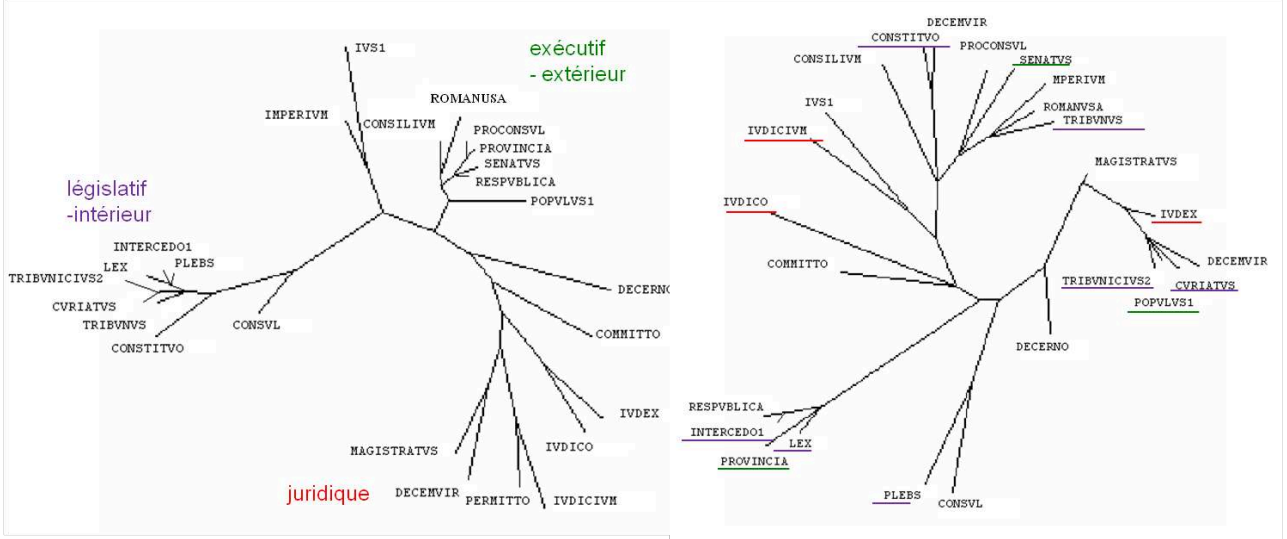

Iudex, iudicium et iudico se retrouvent sur trois branches différentes sensiblement éloignées les unes des autres. De même pour la province (prouincia) qui se sépare du sénat (senatus) qui en a la charge ou pour ius et imperium. Même le tribun de la plèbe voit les deux formants de son nom positionnés à l'opposé l'un de l'autre.

On obtient des résultats assez similaires, quoique moins spectaculaires, pour les formes potestas et potestatem. Dans tous les cas de figure, le pôle législatif intérieur autour de l'élaboration des lois et de l'intercession des tribuns de la plèbe, qui conjoint regroupement thématique et association phraséologique, reste assez stable. Les arbres des énergies des réseaux cooccurrentiels calculés autour des formes potestas et potestatem ont de ce fait un certain air de ressemblance avec l'arbre des énergies du réseau cooccurrentiel calculé autour du lemme POTESTAS, ce qui pourrait certes paraître contraire à nos conclusions. Mais, comme on peut le constater sur la figure 5 , des déplacements apparemment peu nombreux arrivent cependant à déstructurer les regroupements thématiques de l'arbre. Si, comme nous l'avons annoncé, le pôle législatif intérieur reste cohérent, les deux autres regroupements thématiques se dispersent sur l'arbre des énergies calculé autour de la forme potestatem : l'éclatement du pôle juridique est marqué par la séparation de magistratus d'un côté, de decemuir d'un autre, de iudicium et permitto d'un troisième. On retrouve également la séparation, d'une part entre prouincia et senatus (rappelons que c'est le sénat qui attribue les provinces), d'autre part entre ius et imperium (éclatement du pôle exécutif extérieur). Le lemme proconsul, lui, n'apparaît plus sur cet arbre car il n'apparaît jamais dans l'environnement de potestatem et à donc être dû supprimé du réseau cooccurrentiel pour permettre la construction de l'arbre. 
Arbre des énergies du réseau cooccurrentiel calculé autour du lemme POTESTAS (reprise de la figure 2)

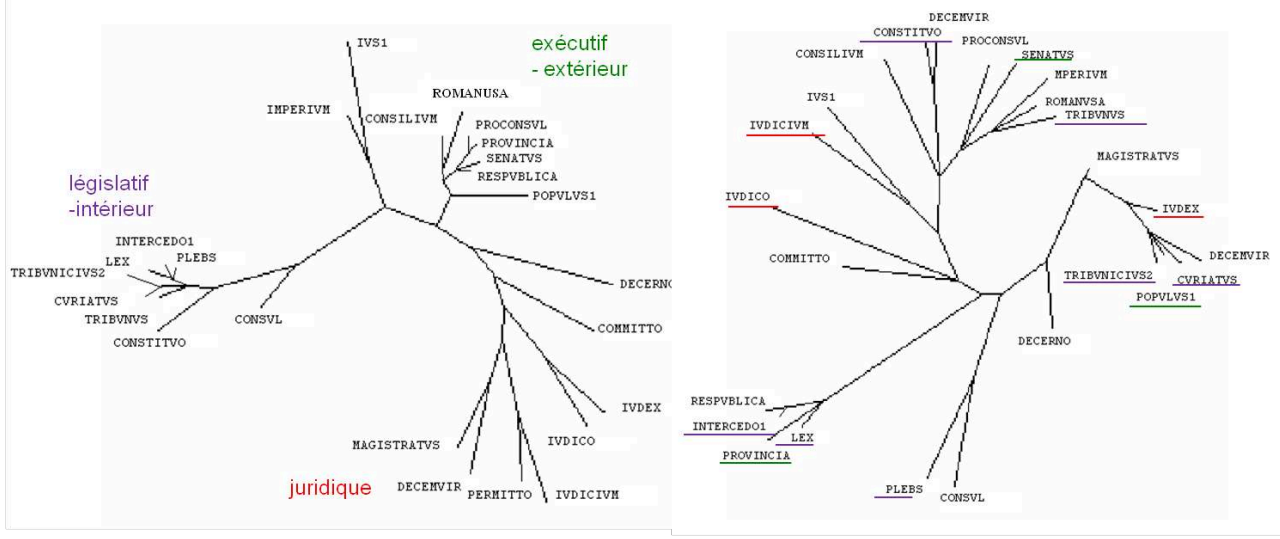

Figure 5. Arbre des énergies du même réseau cooccurrentiel calculé autour de la forme potestatem

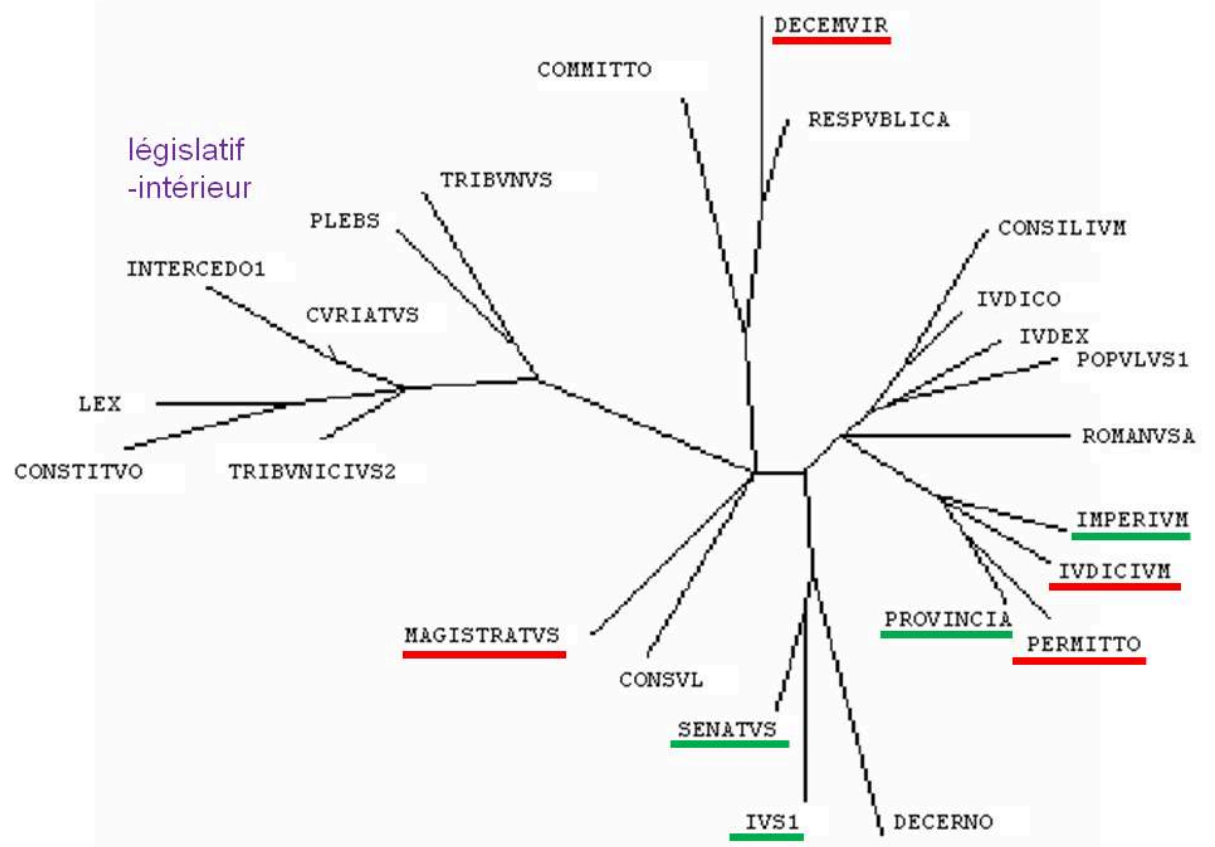

Des éclatements similaires apparaissent sur l'arbre des énergies du même réseau cooccurrentiel calculé autour de la forme de nominatif potestas, dont a été exclu proconsul toujours pour les mêmes raisons (figure 6, ci-dessous).

Ces résultats tendent à confirmer que les arbres des énergies sont vraiment représentatifs de la contextualisation discursive des termes du réseau cooccurrentiel: ces arbres deviennent donc très mal structurés, et difficilement interprétables, lorsqu'une liste de cooccurrents est empruntée à un contexte (celui du lemme) et injectée dans un contexte légèrement différent (celui de la forme); en revanche, les arbres des disponibilités restent interprétables dans la mesure où ils donnent à voir des rapprochements plus décontextualisés et éventuellement stabilisés dans le lexique de la langue. 
Figure 6. Arbre des énergies du même réseau cooccurrentiel calculé autour de la forme potestas

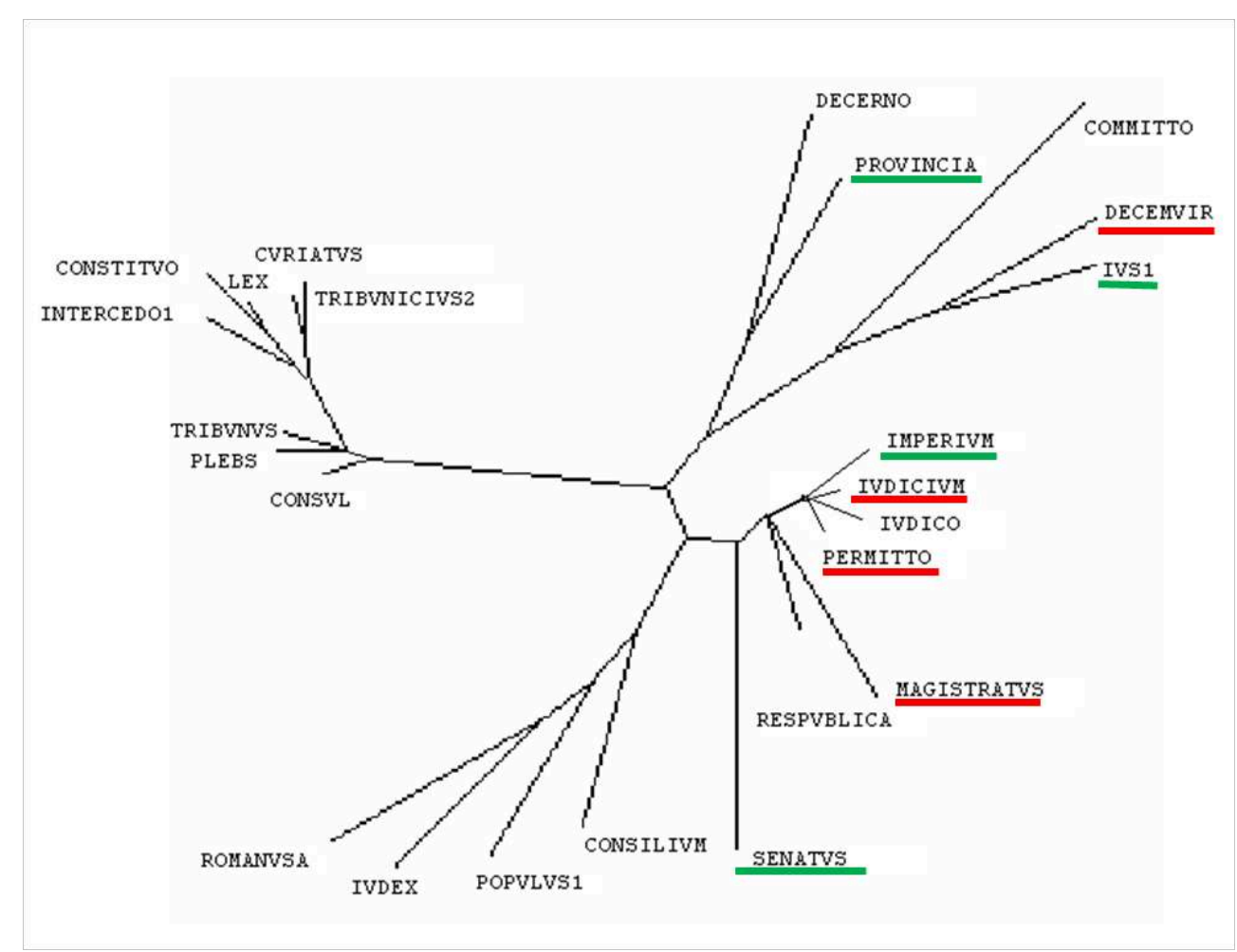

\subsection{Les réseaux cooccurrentiels du lemme RATIO et de ses formes : impact de la polysémie}

Le deuxième exemple que nous avons choisi est celui du lemme RATIO, dont nous avons signalé la large polysémie ( $(1)$. Les valeurs d'emploi de ce terme sont donc extrêmement variées, et cette diversité s'adosse en partie aux variations morphologiques de la flexion casuelle. Ainsi, les emplois comme nom-support avec évidement sémantique ("façon", "manière ») sont-ils principalement attestés à l'ablatif, pour l'expression des circonstants de manière. A l'inverse, le sens très concret de "compte(s) » apparaît plutôt dans des constructions accusatives après des verbes transitifs tels que « rendre » ou « tenir ».

41 L'hypothèse formulée ici, sur la base des résultats précédents, et que nous allons vérifier dans cette dernière partie, est que l'extrême polysémie du terme devrait susciter un réseau de cooccurrents très diversifiés, et que l'abstraction de la lemmatisation - qui nous permet de rassembler dans un même sous-corpus tous les paragraphes contenant une occurrence du lemme RATIO, quelle que soit sa forme fléchie, et d'y dénombrer tous les lemmes cooccurrents de RATIO - risque de produire un ensemble de données hétérogènes, difficile à structurer et à interpréter, surtout lors de la construction de l'arbre des énergies, c'est-à-dire de celui qui met en évidence les proximités de comportement cooccurrentiel en lien avec les dynamiques discursives et thématiques. A contrario, le réseau des cooccurrents d'une seule forme casuelle devrait permettre de construire une matrice plus cohérente donnant lieu à une analyse et une représentation arborée plus facilement interprétable. 
42 2.3.1 Voyons donc si les données confirment ou infirment nos hypothèses. La figure 7 donne à voir l'arbre des proximités et distances de comportement cooccurrentiel, en termes d'énergie, entre les 36 cooccurrents principaux du lemme RATIO

Figure 7. Arbre des énergies du réseau cooccurrentiel du lemme RATIO

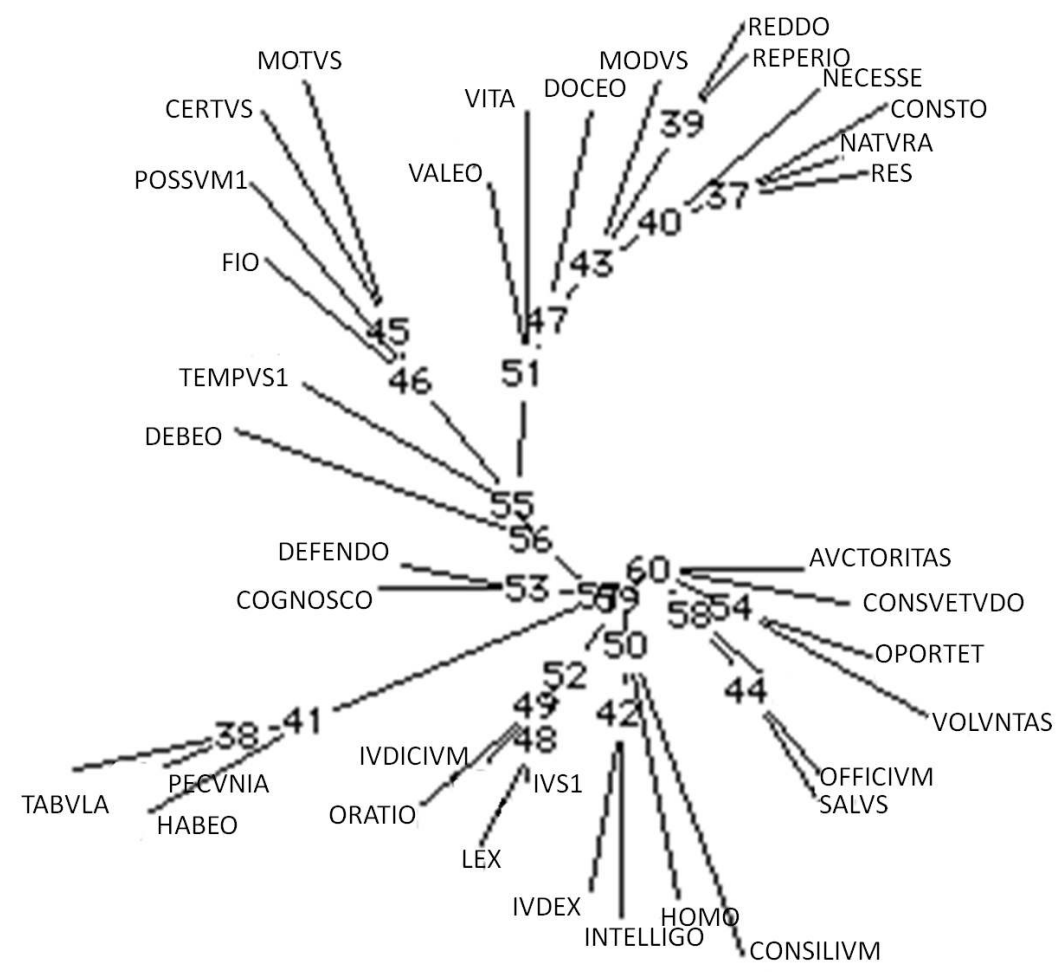

Cet arbre, avec sa forme étoilée, est à l'évidence mal structuré ; aucune ligne de partage ne s'impose de manière évidente pour créer des bipartitions interprétables. Les regroupements thématiques ne sont sans doute pas totalement inexistants, mais restent flous et lâches. Enfin, les taux d'agrégation des premiers nœuds sont relativement faibles ${ }^{7}$.

L'arbre des disponibilités, a priori moins soumis à la contextualisation discursive des cooccurrences, échappe-t-il à cet impact négatif de la polysémie du lemme pivot ? Pas vraiment. Et ses taux d'agrégation ne sont pas bons non plus, aucun n'atteint la valeur de 0,99. 
Figure 8. Arbre des disponibilités du réseau cooccurrentiel du lemme RATIO

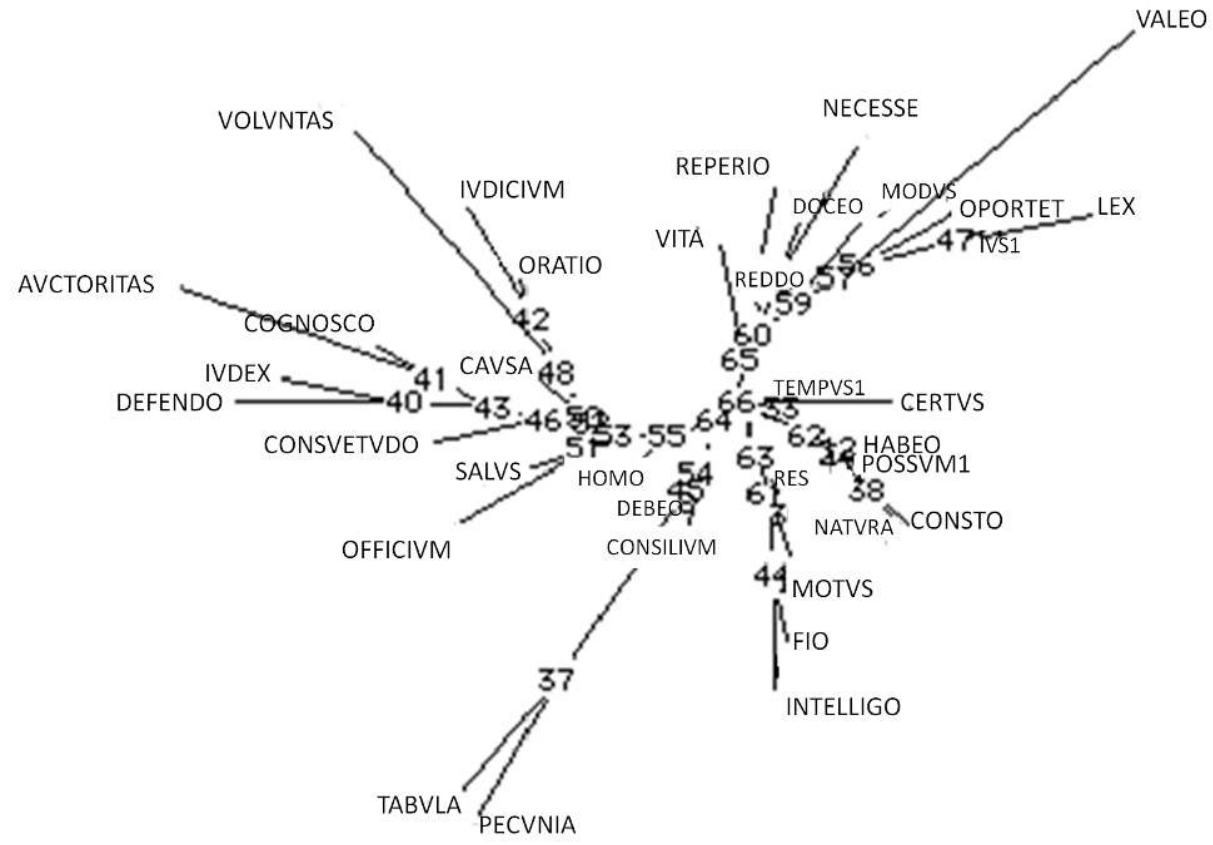

Ce qui frappe, en revanche, c'est que les regroupements proposés par l'algorithme n'aboutissent qu'à former des paires de mots qui, certes, ne forment pas des segments phraséologiques à proprement parler, mais dont certaines pourraient néanmoins correspondre à des expressions récurrentes, comme les verbes fio et intellego (regroupés sur le nœud 44 en bas de l'arbre) et que le retour au texte fait apparaitre dans les séquences suivantes :

Id si fieret intellegebat ( il comprenait que, si cela se faisait... »

Ab se fieri intellegebat ( il comprenait que cela était fait par lui »)

Necessario fieri intellegat ( il comprenait qu'il se faisait nécessairement que... »)

Fieri non posse intellego ( je comprends qu il ne peut pas se faire que...»)

Effice ut quomodo fiat intellegam («Fais en sorte que je comprenne comment il se fait que... »)

En somme, l'impact de la polysémie du lemme est tel ici que son réseau cooccurrentiel, appréhendé dans l'ensemble de la base latine, donc sans filtrage de genre, d'auteur ou d'époque, est très difficile à structurer et que la différence entre énergies et disponibilités s'en trouve minimisée.

2.3.2 L'analyse des cooccurrents d'une seule forme fléchie va-t-elle nous permettre de retrouver une meilleure structuration des arbres? Voici l'arbre des énergies, puis celui des disponibilités, du réseau cooccurrentiel des 24 termes de sens plein les plus significativement attirés par la forme d'ablatif ratione. 
Figure 9. Arbre des énergies du réseau cooccurrentiel de la forme ratione

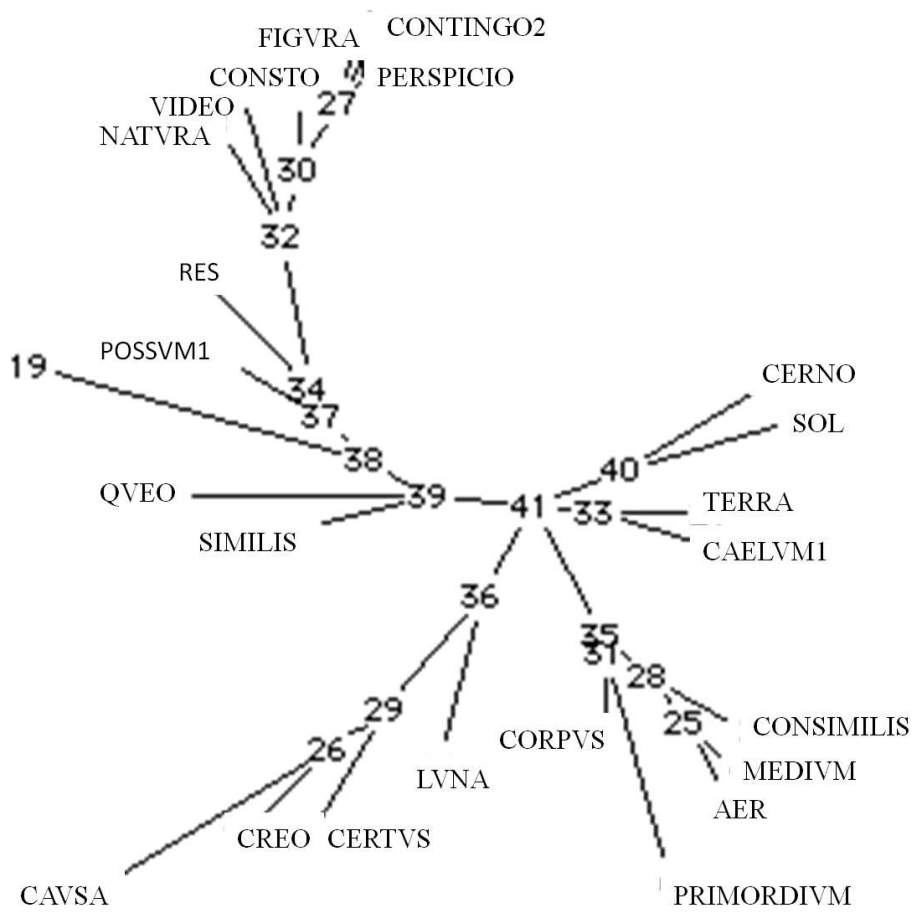

Figure 10. Arbre des disponibilités du réseau cooccurrentiel de la forme ratione

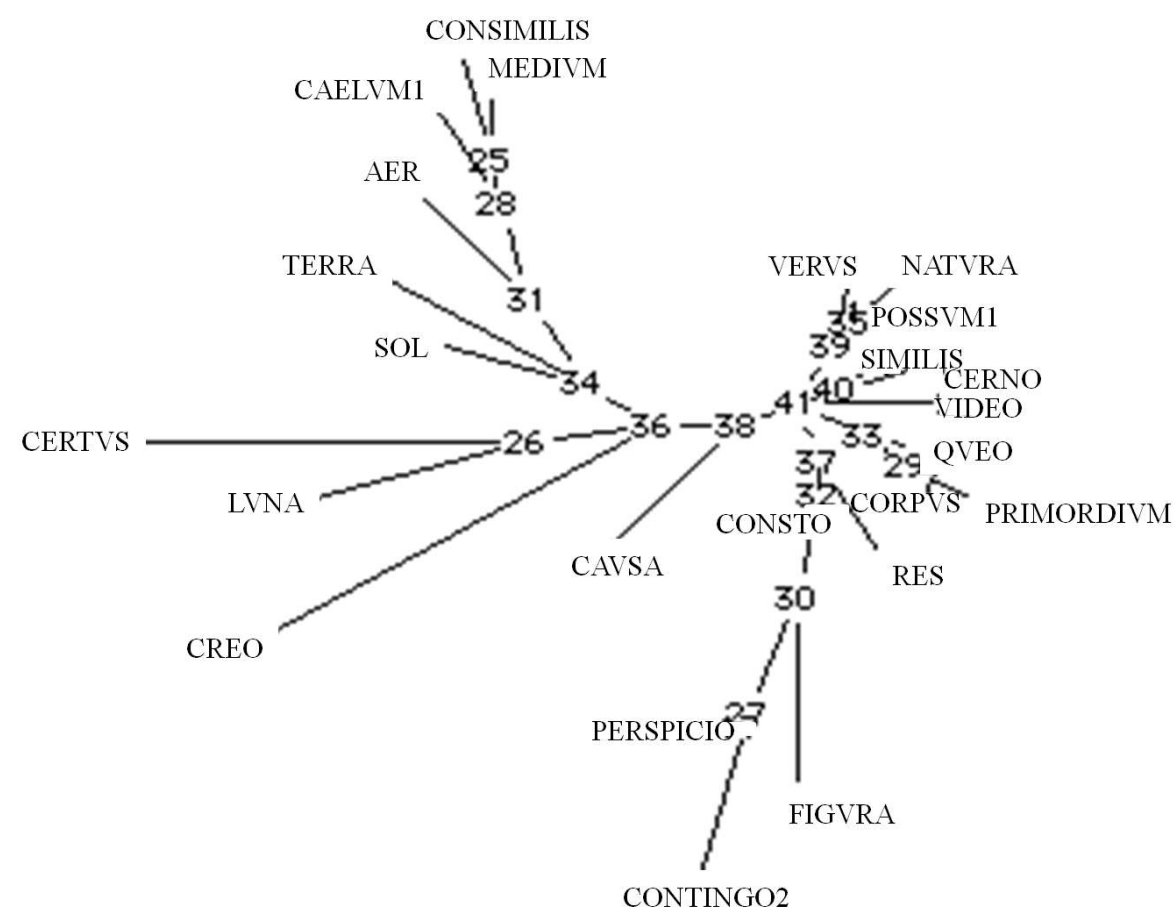

Cette fois-ci, l'arbre des énergies (figure 9), offre une bipartition centrale assez claire (entre le nœud 39 et le nœud 41) et deux regroupements thématiques faciles à 
interpréter pour le latiniste: la partie ouest/nord-ouest de l'arbre rassemble un vocabulaire abstrait, que l'on rencontre notamment dans la prose cicéronnienne ; la partie est / sud-est rassemble, elle, un vocabulaire plus concret, caractéristique de la poésie. Le vocabulaire propre à Lucrèce, qui philosophe en vers et dont l'impact est non-négligeable dans ce sous-corpus constitué autour de la forme ratione, se répartit de manière logique sur les deux parties de l'arbre (figura, contingo, queo avec les mots abstraits, creo, consimilis et primordium avec les mots concrets).

Il est très intéressant de noter la place qui est réservée, dans l'arbre des énergies, aux deux verbes cerno et perspicio, qu'on peut tous les deux traduire, en première approximation, par « voir, percevoir ». Perspicio est dans le groupe des mots abstraits : volontiers employé par Cicéron, il exprime, dans le contexte immédiat de ratione, une perception intellectuelle ou associée à un processus de compréhension; il peut régir une proposition complétive, à l'infinitif ou interrogative indirecte. Cerno, en revanche, est dans le groupe des mots concrets : dans le même contexte, il consacre donc une part importante de ses effectifs à entrer en cooccurrence avec les 23 autres termes du réseau de la même manière que le font les mots concrets que sont sol (« le soleil»), terra (« la terre») ou caelum («le ciel»). Et de fait, le retour aux textes atteste un emploi majoritaire chez Lucrèce dans des expressions comme cernere et audire («voir et entendre »), cernere ante oculos (« avoir devant les yeux »), cernere per oculos («voir de ses yeux »), nostri cernere sensus nequeunt (" nos sens ne peuvent pas percevoir »), etc.; en revanche, aucune construction complétive n'est attestée.

Or dans l'arbre des disponibilités (figure 10), cette belle distinction s'estompe : cerno rejoint le groupe des mots abstraits (cette fois-ci situé dans la partie droite de l'arbre), avec l'ensemble des verbes à l'exception de creo (« créer ») - d'ailleurs très mal rattaché à l'ensemble de l'arbre. Alors que, dans la dynamique discursive qui lui est propre, le verbe cerno contextualise l'environnement de la forme ratione comme le ferait un verbe concret (c'est-à-dire consacre une bonne proportion de ses effectifs à des associations au sémantisme concret), sa disponibilité, elle, (c'est-à-dire l'ensemble des associations qu'il reçoit des autres termes) le ramène vers la catégorie abstraite des verbes et concepts généraux. De la même façon, corpus («corps») et primordium («origine ») quittent la zone des mots poétiques concrets pour rejoindre celle des mots plus abstraits et à connotation philosophique, ce qui n'a, sémantiquement, rien de bien surprenant, et correspond au sens général de ces deux mots en langue.

51 Ce test sur les cooccurrents du lemme RATIO et de l'une de ses formes fléchies nous apporte donc confirmation qu'en cas de polysémie marquée, la structuration du réseau cooccurrentiel d'une forme est plus cohérente que celle du réseau cooccurrentiel du lemme. Il nous montre aussi que cette cohérence forte autour de la forme induit une assez grande stabilité entre l'arbre des énergies et celui des disponibilités. Certes, quelques changements ont lieu de l'un à l'autre, mais ils sont peu nombreux. Le filtre imposé par la forme fléchie au contexte semble donc, non seulement assurer la cohérence du réseau cooccurrentiel, mais également réduire les effets généralement observés de l'asymétrie de la cooccurrence et resserrer plus étroitement les liens entre contraintes lexicales stabilisées en langue d'une part et structuration thématique en discours d'autre part. 


\section{Conclusion}

De tout ceci, on peut tirer quelques conclusions. Tout d'abord, pour interpréter la construction du sens en contexte à partir des cooccurrents d'un mot, faire abstraction de la fonction syntaxique de ce mot n'est pas anodin : dans une analyse fine joignant quantitatif et qualitatif, pour appréhender le rôle de la cooccurrence dans la construction du sens en contexte, il serait préférable, en latin, de travailler sur les réseaux cooccurrentiels de chacune des formes plutôt que sur celui du lemme. La chose méritera d'être vérifiée avec d'autres réseaux, et il s'agira aussi de tester si elle se vérifie pour d'autres langues.

Ensuite, le test paradoxal auquel nous avons soumis ici les données autour du lemme POTESTAS tend à confirmer la validité d'un modèle asymétrique de la cooccurrence. Cette asymétrie entre disponibilité et énergie est sous-tendue par une opposition entre des affinités stabilisées dans les ressources lexicales (relevant de la phraséologie et de l'étymologie) et des affinités en discours autour de champs thématiques et notionnels (même si l'expression de ces derniers passe évidemment par l'exploitation des ressources lexicales préconstruites). Ce résultat reste cependant à affiner dans la mesure où, nous venons de le voir avec les données autour de ratione, dans un réseau fortement cohérent, la thématique discursive et le «prêt à parler » lexical convergent fortement.

\section{BIBLIOGRAPHIE}

Barthélemy J.-P. \& Luong X. (1998). « Représenter les données textuelles par des arbres », in Brunet E. \& Mellet S. (éd.), Actes des 4èmes JADT, Université de Nice : UMR 6039, 49-70.

Heiden S. \& Lafon P. (1998). « Cooccurrences. La CFDT de 1973 à 1992 », Des mots en liberté, Mélanges Maurice Tournier, ENS Editions, tome 1, 65-83.

Luong X., Brunet E., Longrée D., Mayaffre D., Mellet S. \& Poudat C. (2010). « La coocurrence, une relation asymétrique? ", in S. Bolasco, I. Chiari \& L. Giuliano (éd.), Statistical Analysis of Textual Data, Proceedings of the 10th International Conference Journées d'Analyse statistique des Données Textuelles, 9-11 June 2010, Sapienza University of Rome, Rome, LED, 321-332.

Mayaffre D. (2008). « De l'occurrence à l'isotopie. Les co-occurrences en lexicométrie », Sémantique \& Syntaxe $9: 53-72$.

\section{ANNEXES}

Formes cooccurrentes spécifiques de la forme de nominatif ratio

\begin{tabular}{|l|l|l|l|}
\hline écart & corpus & texte & mot \\
\hline
\end{tabular}




\begin{tabular}{|c|c|c|c|}
\hline 37.58 & 456 & 456 & ratio \\
\hline 11.41 & 18680 & 282 & est \\
\hline 9.00 & 40 & 13 & habenda \\
\hline 7.17 & 62 & 11 & perfecta \\
\hline 6.63 & 866 & 29 & natura \\
\hline 6.18 & 467 & 20 & ratione \\
\hline 5.45 & 846 & 24 & eadem \\
\hline 5.32 & 749 & 22 & alia \\
\hline 5.25 & 8196 & 105 & quae \\
\hline 5.20 & 146 & 10 & propterea \\
\hline 5.16 & 3253 & 53 & sit \\
\hline 4.76 & 231 & 11 & solis \\
\hline 4.74 & 49 & 6 & defensionis \\
\hline 4.72 & 1194 & 26 & rerum \\
\hline 4.71 & 13 & 4 & radii \\
\hline 4.68 & 113 & 8 & dicendi \\
\hline 4.67 & 1935 & 35 & autem \\
\hline 4.48 & 4329 & 60 & enim \\
\hline 4.45 & 2118 & 36 & qua \\
\hline 4.35 & 101 & 7 & illinc \\
\hline 4.30 & 346 & 12 & aliqua \\
\hline 4.30 & 238 & 10 & naturae \\
\hline 4.27 & 21 & 4 & exponere \\
\hline 4.24 & 44 & 5 & constet \\
\hline 4.21 & 45 & 5 & habita \\
\hline 4.20 & 1458 & 27 & omnis \\
\hline
\end{tabular}




\begin{tabular}{|l|l|l|l|}
\hline 4.09 & 82 & 6 & consilii \\
\hline 4.08 & 83 & 6 & necessest \\
\hline 4.05 & 725 & 17 & denique \\
\hline 4.05 & 653 & 16 & uitae \\
\hline 4.05 & 85 & 6 & consuetudo \\
\hline
\end{tabular}

Lemmes cooccurrents spécifiques de la forme de nominatif ratio

\begin{tabular}{|l|l|l|l|}
\hline écart & corpus & texte & mot \\
\hline & & & \\
\hline 37.58 & 1403 & 495 & ratio \\
\hline 9.48 & 39989 & 465 & svm1 \\
\hline 9.19 & 1392 & 50 & natvra \\
\hline 6.87 & 6671 & 104 & res \\
\hline 6.64 & 108 & 12 & perfectvs2 \\
\hline 5.17 & 147 & 10 & propterea \\
\hline 5.05 & 9 & 4 & absinthivm \\
\hline 4.95 & 10 & 4 & contingo2 \\
\hline 4.89 & 717 & 20 & officivm \\
\hline 4.81 & 429 & 15 & consto \\
\hline 4.77 & 228 & 11 & rectvs \\
\hline 4.72 & 3206 & 50 & cavsa \\
\hline 4.64 & 1936 & 35 & avtem \\
\hline 4.55 & 475 & 15 & sol \\
\hline 4.40 & 328 & 12 & cvm1 \\
\hline 4.36 & 1307 & 26 & bonvm \\
\hline 4.35 & 230 & 10 & defensio \\
\hline 4.34 & 4300 & 59 & enim2 \\
\hline
\end{tabular}




\begin{tabular}{|l|l|l|l|}
\hline 4.28 & 105 & 7 & illinc \\
\hline 4.25 & 193 & 9 & affectvs1 \\
\hline 4.17 & 9856 & 111 & omnis \\
\hline 4.04 & 20415 & 203 & non \\
\hline 4.04 & 449 & 13 & postvlo \\
\hline 4.03 & 725 & 17 & deniqve \\
\hline 4.03 & 10 & 3 & valesco \\
\hline
\end{tabular}

Formes cooccurrentes spécifiques de la forme d'accusatif rationem

\begin{tabular}{|l|l|l|l|}
\hline écart & corpus & texte & Mot \\
\hline & & & \\
\hline 37.58 & 293 & 293 & Rationem \\
\hline 6.99 & 63 & 10 & Offici \\
\hline 6.82 & 156 & 13 & Reddere \\
\hline 6.03 & 10 & 5 & Reddendam \\
\hline 5.38 & 11009 & 111 & Ad \\
\hline 5.25 & 7364 & 81 & Esse \\
\hline 5.12 & 24 & 5 & Propraetore \\
\hline 4.77 & 1214 & 23 & Hanc \\
\hline 4.75 & 10735 & 103 & Qui \\
\hline 4.69 & 573 & 15 & Belli \\
\hline 4.66 & 17 & 4 & Habendam \\
\hline 4.61 & 39 & 5 & Humanitatis \\
\hline 4.50 & 114 & 7 & Frumenti \\
\hline 4.48 & 13815 & 123 & Cum \\
\hline 4.43 & 411 & 12 & Mei \\
\hline 4.40 & 985 & 19 & C \\
\hline
\end{tabular}




\begin{tabular}{|l|l|l|l|}
\hline 4.37 & 49 & 5 & Defensionis \\
\hline 4.36 & 8 & 3 & Adhibe \\
\hline 4.32 & 130 & 7 & Facti \\
\hline 4.30 & 132 & 7 & Diligenter \\
\hline 4.24 & 27 & 4 & Legioni \\
\hline 4.19 & 10 & 3 & Gallici \\
\hline 4.14 & 100 & 6 & Tantae \\
\hline 4.11 & 62 & 5 & mitto \\
\hline 4.09 & 104 & 6 & haberi \\
\hline 4.09 & 63 & 5 & oporteret \\
\hline 4.08 & 644 & 14 & habere \\
\hline 4.04 & 157 & 7 & aliquam \\
\hline
\end{tabular}

Lemmes cooccurrents spécifiques de la forme d'accusatif rationem

\begin{tabular}{|l|l|l|l|}
\hline écart & corpus & texte & mot \\
\hline & & & \\
\hline 37.58 & 1403 & 316 & ratio \\
\hline 8.41 & 1133 & 37 & reddo \\
\hline 7.51 & 5204 & 79 & habeo \\
\hline$<p$ class="annex & \multicolumn{3}{|l}{} \\
\cline { 1 - 3 } & & &
\end{tabular}

\section{NOTES}

1. L'abstraction de la lemmatisation permet, en latin, de regrouper sous une même entrée les formes d'un lemme réparties entre 6 cas et 2 nombres pour les substantifs, auxquels il faut ajouter trois genres pour les adjectifs.

2. Les indices 1 ou 2 qui sont parfois accolés aux lemmes servent à désambiguïser des homonymes.

3. Pour le détail, voir les annexes.

4. «Le tribun de la plèbe a posé son véto », « la loi curiate », « la loi tribunicienne ».

5. "Le sénat a décidé et le peuple a ordonné », « il a été décidé par le sénat que », " les provinces sont attribuées [aux proconsuls]».

6. «Juger, juge, jugement». 
7. Les taux sont de 0,96 pour le premier et pour le sixième nœuds, 0,97 pour le deuxième, 0,92 pour le septième, 0,95 pour les six suivants, puis 0,74 , etc.; seuls les troisième, quatrième et cinquième nœuds (respectivement $n^{\circ} 38,39$ et 40 sur la figure) ont un taux proche de ou supérieur à 0,99 ; ils regroupent respectivement les termes consto, natura et res (thématique probablement lucrécienne), tabula et pecunia (domaine économique) et enfin les deux verbes reddo et reperio.

\section{RÉSUMÉS}

Cet article essaye d'évaluer l'impact de la lemmatisation, ou, inversement, de la flexion casuelle sur les réseaux cooccurrentiels d'un mot-pôle en latin. Conjointement, il exploite cet impact pour approfondir l'examen de l'asymétrie des relations de cooccurrence.

Nos précédents articles méthodologiques, consacrés notamment à l'asymétrie de la cooccurrence, reposaient en effet sur le dénombrement des cooccurrents d'un mot-pôle considéré et décompté sous sa forme de lemme. Or, si la cooccurrence est bien la "forme minimale du contexte " [Mayaffre 2008] qui contribue à construire le sens textuel, la question se pose alors de la pertinence de l'abstraction opérée par la lemmatisation. Les différentes formes fléchies d'un mot en latin sont porteuses non seulement des catégories du nombre (et, pour les adjectifs et les pronoms, du genre), mais aussi de marques casuelles étroitement reliées à la fonction syntaxique et l'ensemble est susceptible d'avoir une influence sur le contexte immédiat du mot, y compris dans sa dimension thématique.

Nous montrons donc dans un premier temps que la forme casuelle d'un mot latin détermine pour une bonne part la liste de ses cooccurrents spécifiques. Nous exploitons ensuite ce constat pour confirmer, au moyen de deux tests différents qui utilisent le réseau cooccurrentiel des diverses formes fléchies d'un même lemme, une hypothèse précédemment avancée [Luong et al. 2010] selon laquelle l'asymétrie des cooccurrences est, pour partie au moins, une image de la double insertion d'une forme, d'une part, dans un paradigme lexico-sémantique stabilisée en langue, d'autre part, dans un réseau de relations syntagmatiques actualisées en discours.

Cooccurrential Asymmetry and Contextualization

This papers aims to evaluate the impact of the lemmatization, or, on the other way round, of the casual inflection on co-occurrence networks organized around a word-pole in Latin. At the same time, it will provide new insights into the asymmetrical relations of co-occurrence.

Our previous papers on this subject took only into account co-occurrence networks of lemmas. Now, if the co-occurrence indeed is the "minimal form of the context" [Mayaffre 2008] which contributes to build textual meaning, one can wonder if the abstraction operated by the lemmatization is appropriate in this case. The various inflected forms of a Latin word signify not only the number (and, for adjectives and pronouns the gender), but also the case which is closely connected to the syntactic function. This could have an influence on the immediate context of the word, regarding also its thematic dimension.

At first, we show that the case of a Latin word conditions largely the list of its specific collocates. By leaning on this observation and on two different tests based on the study of co-occurrence networks of the various inflected forms of the same lemma, we confirm our previous hypothesis [Luong et al. 2010]: the asymmetry of the co-occurrences reflects, at least partially, the double 
insertion of a word, on one hand, in a lexico-semantic paradigm stabilized in language, on the other hand, in a network of syntagmatic relations realized in speech.

INDEX

Mots-clés : cooccurrence, lemmatisation, flexion casuelle, contextualisation, relations syntagmatiques

Keywords : co-occurrence, lemmatization, inflected forms, contextualization, syntagmatic relations

\section{AUTEURS}

\section{DOMINIQUE LONGRÉE}

LASLA, Université de Liège et FUSL (Bruxelles), Belgique

\section{SYLVIE MELLET}

Université Nice Sophia Antipolis, CNRS, BCL, UMR 7230, Nice , France 